\title{
INMIGRANTES EMPRENDEDORES DURANTE LA CRISIS ECONÓMICA. ESTUDIO DE CASOS EN LA CIUDAD DE MADRID
}

\section{IMMIGRANT ENTREPRENEURS DURING THE ECONOMIC CRISIS. CASE STUDIES IN MADRID CITY}

Juan Antonio Cebrián de Miguel*, Beatriz Cristina Jiménez Blasco**

y Rosa María Resino García***

Resumen: Los inmigrantes emprendedores han tenido que adaptar sus negocios o, incluso, abandonarlos, debido al impacto de la crisis económica que ha afectado de forma muy especial al consumo y, por tanto, a la actividad comercial en general. Para apreciar los cambios habidos en esta etapa se han seleccionado seis ejes urbanos de la ciudad de Madrid, con presencia significativa de negocios de titularidad extranjera, y que los autores habían estudiado en el año 2009. Y se han vuelto a visitar y analizar en 2015. Actualmente, el llamado empresariado étnico, para mantener sus negocios, tiende a captar una clientela más amplia, abandonando una excesiva especialización y mejorando la presencia de los establecimientos comerciales y de los productos y servicios que ofrece.

Científico Titular del Instituto de Economía, Geografía y Demografía del Consejo Superior de Investigaciones Científicas.

juanantonio.cebrian@cchs.csic.es

** Grupo de Investigación Geografías de la inmigración en la Comunidad de Madrid. Inmigración y Sistemas Productivos, UCM 930090

Departamento de Geografía Humana - Universidad Complutense de Madrid. bcjimene@ghis.ucm.es

**** Grupo de Investigación Geografías de la inmigración en la Comunidad de Madrid. Inmigración y Sistemas Productivos, UCM 930090

Departamento de Geografía Humana - Universidad Complutense de Madrid. r.resino@ghis.ucm.es 
Palabras clave: Madrid, inmigración, empresariado étnico, crisis económica.

Abstract: Immigrant entrepreneurs have had to adapt or even abandon their businesses due to the impact of the economic crisis that affected consumption especially and, therefore, business activity in general. In order to appreciate the changes at this stage of significant impact of the crisis, six urban axes of the city of Madrid have been selected that have a significant presence of foreign-owned shops and services, which were studied by the authors in 2009. They were visited and analyzed again in 2015. Currently, to maintain their businesses, these so-called ethnic entrepreneurs tend to attract a wider customer base, abandoning excessive specialization while in its place improving the presence of commercial establishments and the products and services offered.

Keywords: Madrid, immigration, ethnic entrepreneurship, economic crisis.

\section{INTRODUCCIÓN}

La faceta empresarial de los inmigrantes en los países occidentales desde el último cuarto del siglo XX despertó un pronto interés entre los estudiosos de la inmigración, quienes acuñaron términos, como empresas étnicas, negocios étnicos, comercio étnico, empresas circuito, etc. (Evans, 1989; Portes et al., 2003).

En el caso de España, uno de los efectos más palpables de la intensiva inmigración experimentada desde finales de la década de los ochenta ha sido la proliferación de pequeñas empresas y comercios establecidos por inmigrantes en sus ciudades más importantes (Aramburu, 2002; Trinidad, 2003; Arjona y Checa, 2006; Solé, Parella y Cavalcanti, 2007; Pagliarin, 2011; Miret y Serra, 2013; Muñoz-Bullón, 2014).

Estas empresas han facilitado la integración de los inmigrantes en la sociedad, convirtiéndose en herramientas para el autoempleo, la promoción socioeconómica y la regeneración de la trama urbana y su tejido social (Aramburu, 2002; Beltrán et al., 2006).

Diversos autores han estudiado las características de estos negocios y su impronta en la ciudad de Madrid (Buckley, 1998; 
Cavalcanti, 2009; Cebrián de Miguel y Bodega, 2002; García Ballesteros et al., 2006; García Ballesteros y Jiménez Blasco, 2012c; Gómez Crespo, 2006).

Este trabajo constituye una etapa más en nuestra línea de investigación sobre el empresariado inmigrante en Madrid. En él se exponen los resultados de una revisión actual de los negocios de inmigrantes en una serie de ejes comerciales madrileños que fueron estudiados en el año 2009 (García Ballesteros et al., 2012a), para observar su evolución durante los años más duros de la crisis económica.

Lógicamente, los cambios experimentados en los negocios de barrio no sólo se deben a los efectos de la citada crisis, sino también a otros factores ligados a las modificaciones en los hábitos de consumo y ocio de la población en general (Cebrián, Jiménez Blasco y Resino, conferencia 2016). Pero, es evidente que la fuerte retracción del consumo, ligada a la crisis económica, ha forzado a muchos emprendedores inmigrantes a modificar sus estrategias comerciales para continuar con sus negocios, o bien a abandonar su trayectoria empresarial y elegir otro modo de vida, e incluso plantearse la vuelta a sus lugares de origen o la emigración a otro destino que ofrezca mejores expectativas (García Ballesteros y Jiménez Blasco, 2014). Por otro lado, no se puede ignorar que, igualmente, el pequeño empresario autóctono está sufriendo los efectos de la crisis y de los cambios sociodemográficos recientes. Es necesario ahondar más en esta línea de investigación, y comparar en posteriores trabajos las diferencias y similitudes entre sus prácticas comerciales y las del empresariado inmigrante.

\section{EL EMPRESARIADO INMIGRANTE: ALGUNAS CLAVES DE INTERPRETACIÓN}

Una de las consecuencias de la fuerte inmigración internacional que se intensificó tras la crisis del petróleo en los años 70 del siglo $\mathrm{XX}$, radicó en el establecimiento de numerosos negocios de inmigrantes en los barrios de las principales ciudades de acogida. Su impacto en las sociedades occidentales constituye un fenómeno que ha recibido distintos nombres -comercio étnico, negocios de inmigrantes,...- y se viene estudiando a partir de la década de los 90 de 
la pasada centuria, desde diversas disciplinas científicas y bajo diferentes concepciones teóricas (Light y Bhachu, 1992; Light y Gold, 2000; Portes y Shafer, 2006).

En este trabajo no entramos en el debate, siempre polémico, sobre las diferencias conceptuales entre los términos empresariado inmigrante y empresariado étnico. Utilizamos la denominación de empresariado inmigrante para referirnos al colectivo de inmigrantes, considerados como categoría social (García Ballesteros et al., 2004), que establece sus negocios con ahorros propios y/o préstamos de familiares y compatriotas, empleándose a sí mismo como la mano de obra básica de su empresa (Light, y Bonacich, 1988; Riesco, 2003). En este sentido, se asemeja a la definición de empresariado étnico que manejan otros autores, como Beltrán, Oso, y Ribas (2006). Se trata, por tanto, de un pequeño empresario, que debemos desligar de la figura del inversor extranjero que puede montar grandes empresas, cadenas comerciales, etc.

También empleamos el término negocio étnico y sus análogos en su sentido amplio, al igual que autores, como Solé y Parella (2005), entre otros, para los que los negocios étnicos son todos aquellos regentados por inmigrantes, independientemente del tipo de productos que ofrezcan y su potencial clientela. No obstante, hay autores que emplean este concepto de forma más precisa, considerando que el negocio étnico es aquel que va dirigido específicamente a colectivos de inmigrantes, especialmente a compatriotas.

La diversidad de tipologías de negocios étnicos con una orientación empresarial muy definida, tratada ampliamente por varios autores (Ambrosini, 1996; Solé y Parella, 2005; Cavalcanti, 2009), ha ido dando paso a una situación en la que se difuminan los límites entre diferentes orientaciones comerciales. Así, el controvertido término de negocio étnico que se comenzó a utilizar solo para aquellos comercios y servicios que tenían una clientela básica de inmigrantes, poco a poco se comenzó a usar de una forma más general (Solé et al., 2007) y bajo dicho término se denominó a todos aquellos negocios regentados por inmigrantes, independientemente del tipo de productos que ofrezcan y su potencial clientela.

Desde un punto de vista teórico, las interpretaciones "culturalistas" sobre el fenómeno, que asociaban la aparición de los negocios étnicos con las necesidad del inmigrante de encontrar un entorno de compatriotas que le permitiera mantener sus hábitos de consumo 
y de estilo de vida de sus países de origen (Wilpert, 2003; Galbraith et al., 2007), fueron cuestionados por otros enfoques teóricos. Pero sigue existiendo una línea de investigación que remarca el papel de los negocios étnicos como forma de preservación de tradiciones e identidades culturales de ciertos colectivos de inmigrantes, cuando se encuentran poco integrados en las sociedades receptoras.

Por otra parte, las denominadas aproximaciones "estructuralistas" explicaron la creación de negocios étnicos como una oportunidad en el mercado de trabajo para inmigrantes que elegían el autoempleo como nicho laboral. Este enfoque pone el énfasis en la estructura de oportunidades que ofrecen las sociedades en que se insertan los colectivos de emprendedores inmigrantes (Garcés, 2011).

La perspectiva "ecológica" ha hecho hincapié en los procesos de sustitución de población residente y de negocios en el espacio urbano, a partir de los flujos inmigratorios internacionales. El progresivo abandono del comercio tradicional, por parte del pequeño empresario autóctono, aparece como un elemento determinante en investigaciones más generales sobre procesos de ocupación de barrios por población inmigrante (Aramburu, 2002; Trinidad, 2003; Arjona y Checa, 2007; Pagliarin, 2011; Miret y Serra, 2013, Torres, 2006; entre otros).

Otrasinterpretaciones teóricas, consideradas como "integradoras" o "interaccionistas", subrayan que el comercio no es una actividad exclusivamente mercantil, sino que posee una dimensión pública, social y económica con incidencias claras en la vida ciudadana y en las relaciones de convivencia. En este sentido se relaciona la proliferación de negocios étnicos tanto con aspectos culturales, como económicos (Kloosterman, 2001). Desde este punto de vista se considera que la apertura de pequeñas empresas por parte de inmigrantes ha tenido un papel significativo, pues han favorecido su integración en la sociedad de acogida y, al mismo tiempo, se han convertido en una herramienta que contribuye a revitalizar barrios envejecidos y deteriorados (Beltrán et al. 2006; Gómez Crespo, 2006; Miret y Serra, 2013).

Con el paso del tiempo los comercios y servicios montados por inmigrantes se han ido consolidando en la cultura del país de acogida (Aramburu, 2002; Wilpert, 2003; Espinosa, 2008, 2011). El exotismo que caracterizó a una buena parte de los primeros negocios étnicos ha ido integrándose en la compra más o menos habitual de la población en general. En este proceso, se considera que el 
empresariado inmigrante ofrece un servicio no sólo comercial, sino también cultural y social, pero también contribuye a un cambio en el territorio, alterando el paisaje comercial, en mayor o menor medida, de muchos sectores urbanos.

La pregunta que muchos investigadores se hicieron en los estudios realizados previos a la crisis (García Ballesteros et al, 2006; Gómez Crespo, 2006) es: ¿cómo los inmigrantes subsistían a partir de unos negocios de barrio que los autóctonos habían ido abandonando por su baja rentabilidad? Varios son los factores explicativos: 1) orientación hacia los colectivos de inmigrantes que buscaban servicios necesarios para ellos y productos de sus países que no encontraban en los comercios españoles, 2) atención a una población con escasa movilidad que había quedado anclada en barrios envejecidos y privada del comercio tradicional que iba desapareciendo, 3) interés por lo exótico de la población en general, 4) autoexplotación con largas jornadas y apertura en días festivos, 5) escasa inversión en los locales, 6) empleo de mano de obra familiar, 7) complementariedad de comercio y servicio (locutorios con venta de productos alóctonos), 8) versatilidad para cambiar rápidamente la orientación comercial si no hay rentabilidad económica suficiente y, sobre todo, 9) el deseo únicamente de unas ganancias para el sostenimiento familiar sin búsqueda de una elevada rentabilidad económica que permitiera altos niveles de vida.

\section{METODOLOGÍA Y FUENTES}

La metodología se basa principalmente en la observación directa de los mismos ejes comerciales de Madrid que analizamos en trabajos anteriores (García Ballesteros y Jiménez Blasco, 2012c). En el trabajo de campo se realiza una cuantificación de los tipos de negocios de inmigrantes existentes y de las nacionalidades de los emprendedores. Así mismo, se lleva a cabo una evaluación más cualitativa de los cambios efectuados para contrarrestar la situación de contracción del consumo que, en general, ha producido la crisis económica.

En esta fase del trabajo se realizan también entrevistas cortas a empresarios inmigrantes y a consumidores para completar las observaciones. En total, se han llevado a cabo unas veinte entrevistas 
a cada colectivo, tanto en el año 2009 como en el 2015, procurando que el número de varones y mujeres fuera similar, e intentando que respondieran personas de distintas edades y diversas nacionalidades.

Las principales preguntas a los empresarios van dirigidas a saber su país de origen, si tienen la nacionalidad española, el tiempo que llevan con el negocio, si han cambiado la actividad del mismo desde que iniciaron su emprendimiento, si han tenido trabajos anteriores. También se les pregunta por las razones de la elección de la localización del negocio, por el tipo de clientes y si solo cuentan con la ayuda familiar para sacar adelante el negocio, o tienen empleados y, en este caso, de qué nacionalidad son.

En cuanto a los consumidores, se intenta averiguar su procedencia y, sobre todo, aproximar la proporción de clientes españoles, frente a la de los inmigrantes. Interesa especialmente valorar, en términos relativos, el número de clientes compatriotas del dueño del establecimiento.

Las cuestiones formuladas a los consumidores en general, inmigrantes y españoles, están orientadas a saber qué productos suelen comprar en los comercios de inmigrantes, si son usuarios de servicios regentados por éstos, la frecuencia con la que acuden a este tipo de establecimientos, y las ventajas o, por el contrario, inconvenientes que observan con respecto a los comercios y servicios de empresarios autóctonos.

Las zonas analizadas durante el año 2009, que han vuelto a visitarse durante el año 2015 para examinar su evolución en lo que a negocios de inmigrantes se refiere, han sido:

- La Avenida de la Albufera, en el distrito del Puente de Vallecas, entre la estaciones de metro de Puente de Vallecas y Portazgo.

- La calle de Alcalá, entre la estaciones de metro de El Carmen y Pueblo Nuevo, en el distrito de Ciudad Lineal.

- La calle del General Ricardos, en el distrito de Carabanchel, entre la Glorieta del Marqués de Vadillo y la estación de metro de Urgel.

- La calle de Bravo Murillo, desde la Glorieta de Cuatro Caminos hasta la estación de metro de Estrecho, en el distrito de Tetuán.

- El Paseo de Extremadura, en el distrito de Latina, entre la Puerta del Ángel y la estación de metro del Alto de Extremadura.

- La calle de Hortaleza, en el distrito Centro.

En el mapa de la Figura 1 puede observarse la localización de 
estos ejes comerciales. Todos ellos coinciden con áreas en las que los inmigrantes residentes tienen una presencia relativamente alta y asimismo son zonas con proporciones medias y altas de extranjeros afiliados a la Seguridad Social en actividades comerciales (García Ballesteros et al. 2012a)

\section{Figura 1}

\section{LOCALIZACIÓN DE LOS EJES COMERCIALES ANALIZADOS}

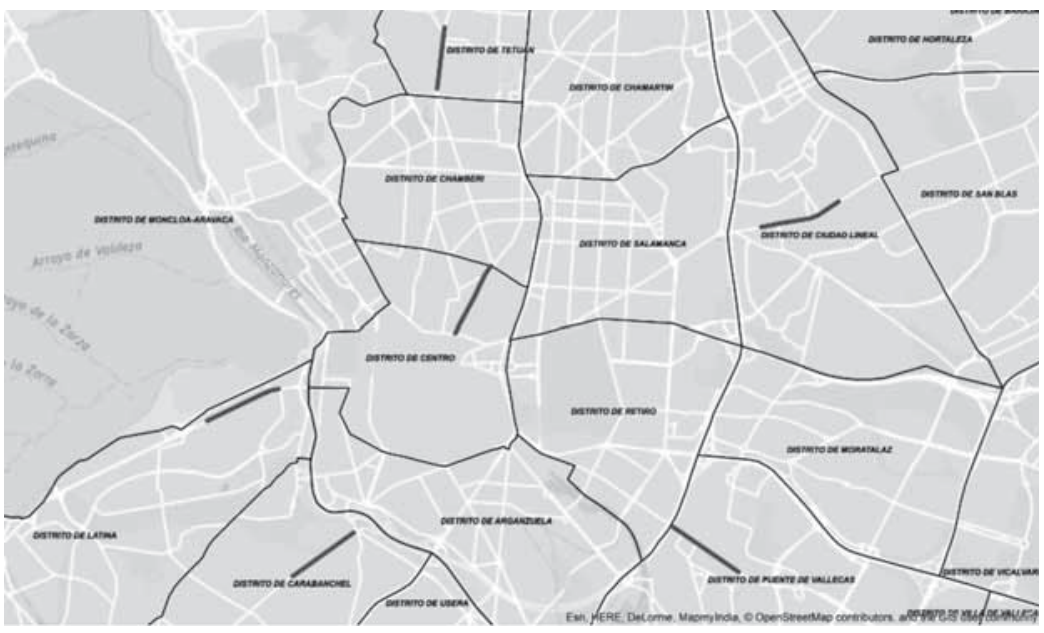

Fuente: elaboración propia.

Las calles seleccionadas articulan sectores urbanos con presencia significativa de comercios y servicios regentados por inmigrantes, que han experimentado un cambio patente en su oferta comercial y, en parte, en su propia fisonomía. Además, en la elección de las calles se han tenido en cuenta una serie de criterios, tales como que los tramos escogidos estén en barrios con una proporción de extranjeros empadronados superior al 15\% y que atraviesen zonas donde el comercio minorista de españoles haya sufrido un claro retroceso, aprovechado por los nuevos emprendedores inmigrantes (García Ballesteros et al. 2006).

Estos ejes comerciales, que podríamos denominar barriales, atravesaron hace unos años un cierto declive debido a la aparición 
de grandes centros comerciales periféricos que ofrecen facilidad de aparcamiento y otros atractivos que sería prolijo detallar aquí (Escudero, 2008). A ello se sumaron los cambios habidos desde hace unos años en el modo de vida y hábitos de consumo de la sociedad. Así como la jubilación de muchos pequeños comerciantes que no se vieron reemplazados por sus hijos, muchos de ellos universitarios y con otras aspiraciones laborales.

Otras fuentes de información utilizadas han sido las Estadísticas publicadas por la Dirección General de Estadística del Ayuntamiento de Madrid y por el Instituto de Estadística de la Comunidad de Madrid. Una fuente estadística muy valiosa fue el Directorio de Unidades de Actividad Económica (DUAE), proporcionada por el antiguo Observatorio Económico del Área de Economía y Empleo del Ayuntamiento de Madrid, para el año 2010, pero que no ha estado disponible en fechas posteriores.

Entre las fuentes bibliográficas, han sido de gran interés las obras que formulan planteamientos teóricos (Light y Bhachu, 1992; Ambrosini, 1996; Light y Gold, 2000; Portes y Shafer, 2006; Beltrán, Oso y Ribas, 2006, entre otros). También han sido una fuente importante de conocimiento los trabajos empíricos realizados en otras ciudades de España (Arjona y Checa, 2005; Parella, 2006) y del mundo (Wilson y Portes, 1980; Cohen, 2003), así como los trabajos sobre determinadas áreas de la ciudad de Madrid (Buckley, 1998; Cebrián y Bodega, 2002; García Ballesteros et al., 2012a), y sobre colectivos específicos de inmigrantes madrileños, como es el caso de su comunidad china (Beltrán y Sáiz, 2009, 2013 y 2015; Tébar, 2010 y 2013).

\section{EVOLUCIÓN DEL EMPRESARIADO INMIGRANTE EN LA ACTIVIDAD COMERCIAL MADRILEÑA DESDE LOS INICIOS DE LA CRISIS}

El comercio en los años previos a la crisis se había convertido en un nicho de empleo muy atractivo para los extranjeros afincados en las ciudades y gran parte de los pueblos de la Comunidad de Madrid. Así, según datos del antiguo Ministerio de Trabajo e Inmigración correspondientes al año 2010, de los 301.803 afiliados extranjeros a la Seguridad Social en el Régimen General en la Comunidad de Madrid, $52.925(17,20 \%)$ estaban en la rúbrica Comercio, de los 
cuales $37.336(71,90 \%)$ eran no comunitarios. Sólo la Hostelería constituía un nicho laboral que incluía un número mayor de afiliados extranjeros en nuestra Comunidad.

Centrándonos en el municipio madrileño, según los datos proporcionados por el Instituto de Estadística de la Comunidad de Madrid, en el año 2008 el porcentaje de afiliados extranjeros a la Seguridad Social en el Régimen General en Comercio se situaba en el 15\%, por detrás de la Construcción y la Hostelería, tal como muestra el Cuadro 1, que nos proporciona una idea de la situación que había en cuanto a la afiliación a la Seguridad Social de extranjeros en la ciudad de Madrid en los inicios de la crisis. La situación en 2016 (Cuadro 1) refleja una baja de 22.497 trabajadores extranjeros en el Régimen General de la ciudad de Madrid y que repercute en descensos en los tres sectores señalados, siendo el descenso más significativo en la Construcción y el más moderado en la Hostelería. El Comercio experimenta un descenso intermedio entre ambos nichos laborales, pero revelador, ya que hay 13.284 trabajadores extranjeros afiliados menos entre el 1 de enero de 2008 y el 1 de enero de 2016. Si bien, hay que tener en cuenta que nos referimos a trabajadores del Régimen General. Información que debemos completar con la obtenida para el Régimen de Autónomos, para su correcta interpretación.

CUADRO 1

AFILIADOS EXTRANJEROS AL RÉGIMEN GENERAL DE LA SEGURIDAD SOCIAL EN LA CIUDAD DE MADRID EN SECTORES ECONÓMICOS ESTRATÉGICOS PARA LOS INMIGRANTES (2008 Y 2016)

\begin{tabular}{|c|c|c|c|c|}
\hline $\begin{array}{c}\text { Actividad } \\
\text { de la Empresa }\end{array}$ & $\begin{array}{c}N^{\circ} \text { de afiliados extranjeros } \\
\text { al Régimen General en la } \\
\text { ciudad de Madrid } \\
\text { (1 enero 2008) }\end{array}$ & $\%$ & $\begin{array}{c}N^{0} \text { de afiliados extranjeros } \\
\text { al Régimen General en la } \\
\text { ciudad de Madrid } \\
\text { (1 enero 2016) }\end{array}$ & $\%$ \\
\hline Construcción & 37.007 & 17,28 & 8.497 & 4,43 \\
\hline Comercio & 32.122 & 15,00 & 18.838 & 9,83 \\
\hline Hostelería & 36.903 & 17,23 & 29.070 & 15,16 \\
\hline Total & 214.204 & $100 \%$ & 191.707 & $100 \%$ \\
\hline
\end{tabular}

Fuente: Instituto de Estadística de la Comunidad de Madrid. 
Los datos de los afiliados extranjeros a la Seguridad Social en el Régimen de Autónomos nos van a aproximar más a nuestro foco de investigación: el llamado "empresariado étnico", aunque no todos ellos se incluyan en la actividad comercial.

En el Cuadro 2 se muestra la evolución de los afiliados extranjeros a la Seguridad Social en el Régimen de Autónomos en la ciudad de Madrid, desde 2007 hasta 2015. Como puede observarse, la evolución de este colectivo, que podemos considerar de emprendedores inmigrantes, fue cayendo desde 2008 hasta 2010, pero a partir de entonces comenzó a mostrar un aumento sostenido, llegando en 2013 a igualar básicamente la situación que había en el año 2009.

CUADRO 2

EVOLUCIÓN DE LOS EXTRANJEROS AFILIADOS A LA SEGURIDAD SOCIAL QUE TRABAJAN EN LA CIUDAD DE MADRID EN EL RÉGIMEN DE AUTÓNOMOS

\begin{tabular}{|c|c|}
\hline Años & $\boldsymbol{N}^{\text {'total }}$ \\
\hline 2007 & 15.404 \\
\hline 2008 & 18.849 \\
\hline 2009 & 18.589 \\
\hline 2010 & 16.417 \\
\hline 2011 & 17.030 \\
\hline 2012 & 18.144 \\
\hline 2013 & 18.651 \\
\hline 2014 & 19.601 \\
\hline 2015 & 20.798 \\
\hline
\end{tabular}

Fuente: Banco de Datos. Ayuntamiento de Madrid.

La comparación de estos dos primeros cuadros es muy significativa, frente al fuerte descenso de los afiliados extranjeros al Régimen General, en el Régimen de Autónomos se ve una evolución positiva a partir del año 2011, lo cual parece indicar que el descenso fuerte de la afiliación de extranjeros a la Seguridad Social se da en el Régimen General, pero no en el de Autónomos, donde los extranjeros se 
dan más de alta, no siempre porque se trate de emprendedores, sino porque de esta manera encuentran trabajo más fácilmente por los menores costes salariales.

En el Cuadro 3 se especifica la evolución de los extranjeros afiliados en el Régimen de Autónomos que trabajan en actividades comerciales en la ciudad de Madrid, pero no se han podido encontrar datos perfectamente comparables para antes del año 2010. Como es lógico, la serie muestra una evolución positiva en consonancia con la que se produce en el Régimen de Autónomos en conjunto, a partir del citado año (Cuadro 2).

\section{CuAdro 3}

EVOLUCIÓN DE LOS EXTRANJEROS AFILIADOS A LA SEGURIDAD SOCIAL EN EL RÉGIMEN DE AUTÓNOMOS, EN EL COMERCIO. (MUNICIPIO DE MADRID)

\begin{tabular}{|c|c|c|}
\hline $\begin{array}{c}\text { AÑOS } \\
\text { (mes de enero) }\end{array}$ & $\begin{array}{c}\text { Trabajan en la ciudad } \\
\text { de Madrid }\end{array}$ & $\begin{array}{c}\text { Residen en la ciudad } \\
\text { de Madrid }\end{array}$ \\
\hline 2010 & 5.674 & 5.431 \\
2011 & 6.130 & 5.855 \\
2012 & 6.739 & 6.408 \\
2013 & 7.200 & 6.860 \\
2014 & 7.622 & 7.407 \\
2015 & 7.905 & 7.719 \\
\hline
\end{tabular}

Fuente: Banco de Datos. Ayuntamiento de Madrid.

Como se desprende del Cuadro 3, los autónomos extranjeros del Comercio que residen en la ciudad de Madrid no son muchos menos que los que trabajan dentro del término municipal, pero están empadronados en otros municipios. Lo que indica que existe una relativa proximidad entre el lugar de trabajo y el de residencia dentro del colectivo que estamos analizando, pues, aunque no son desdeñables las distancias entre los diferentes distritos de nuestra ciudad, los movimientos pendulares de mayor recorrido no parecen excesivos. 


\section{TIPOS DE NEGOCIOS: PRINCIPALES CAMBIOS DETECTADOS ENTRE LOS AÑOS 2009 Y 2015}

Los negocios más emprendidos por inmigrantes se muestran en el Cuadro 4 que refleja las observaciones llevadas a cabo en los ejes comerciales anteriormente citados en dos momentos diferentes en el tiempo: la primera observación realizada en una etapa temprana de la crisis (2009) y una segunda observación en el año 2015, momento en el que la población en general y los inmigrantes en particular llevan ya varios años soportando los efectos de la misma. Sin duda, la recesión económica ha tenido unos efectos palpables en los nichos productivos y en los niveles de empleo de los inmigrantes, detectándose una mayor vulnerabilidad laboral en éstos, conforme la situación de crisis se iba prolongando en el tiempo (Gil-Alonso y Vidal-Coso, 2015).

CuAdRo 4

NEGOCIOS REGENTADOS POR EXTRANJEROS

\begin{tabular}{|l|c|c|}
\hline \multicolumn{1}{|c|}{ Negocios } & $\begin{array}{c}\text { \% sobre el total } \\
\text { 2009 }\end{array}$ & $\begin{array}{c}\text { \% sobre el total } \\
\text { 2015 }\end{array}$ \\
\hline Alimentación & 27 & 30 \\
\hline Hostelería & 20 & 19 \\
\hline Ropa, Calzado y Complementos & 20 & 5 \\
\hline Locutorio/Envío dinero & 11 & 12 \\
\hline Peluquería y Estética & 7 & 4 \\
\hline Bazar & 6 & 2 \\
\hline Telefonía/Informática & 3 & 1 \\
\hline Agencias y Gestorías & 2 & 1 \\
\hline Droguerías, Mercería & 1 & 0 \\
\hline Talleres & 1 & 2 \\
\hline Arreglos (ropa, calzado) & 1 & 3 \\
\hline Otros & 1 & 100 \\
\hline TOTAL & 100 & 21 \\
\hline
\end{tabular}

Fuente: elaboración propia. 
En 2009 casi la mitad de los establecimientos se dedicaban a la venta de productos de alimentación y a la hostelería, este porcentaje ha subido en 4 unidades porcentuales en 2015. Es decir, dos sectores de consumo habitual que se ven afectados menos que otros por las crisis económicas y por la disminución del consumo.

Los empresarios inmigrantes, dentro del sector de la alimentación, muestran una clara preferencia por la apertura de fruterías, en ellas se venden productos más o menos exóticos, pero también las frutas y verduras de mayor consumo en nuestro país (Figura 2). En el año 2009 un buen número de estos establecimientos era regentado por latinoamericanos, en cambio en 2015 éstos han disminuido y observamos un mayor número de empresarios asiáticos, principalmente chinos, iraníes y de países indostánicos. A partir de la observación participante y de información proporcionada por algunos empleados, se constata que algunas fruterías conservan empleados de origen latinoamericano por razones de idioma y afinidad cultural con la clientela autóctona, pero los dueños son asiáticos.

"Esta tienda (frutería) la llevo yo personalmente, pero también tengo un empleado ecuatoriano". (Emprendedor chino)

Las tiendas de alimentación generalistas son mayoritariamente regentadas por la población china, tanto en 2009 como en el momento actual. Su estrategia comercial es prolongar el horario de apertura y especializarse en los productos de más necesidad. Siguen manteniendo productos de uso muy cotidiano (pan, bebidas refrescantes...), y suelen tener algunos productos de consumo oriental (ramen, noodles, fideos de arroz, licores orientales, etc.), cuyo consumo se ha extendido a la población autóctona, especialmente la más joven. Entre 2009 y 2015 algunas de estas tiendas no han variado demasiado, si bien, se observa una tendencia a mejorar su presencia y a modernizarse, así como a uniformar más los productos que venden y elegir las marcas preferidas por la población autóctona.

Los negocios de hostelería han soportado la crisis económica, manteniendo o incluso bajando los precios. En este sector ha ocurrido lo contrario al resto y es que la presencia de la comunidad china ha descendido, frente al aumento de los emprendedores latinoamericanos. 
Figura 2

COMERCIO ÉTNICO EN LA CALLE HORTALEZA DEL DISTRITO CENTRO DE LA CAPITAL

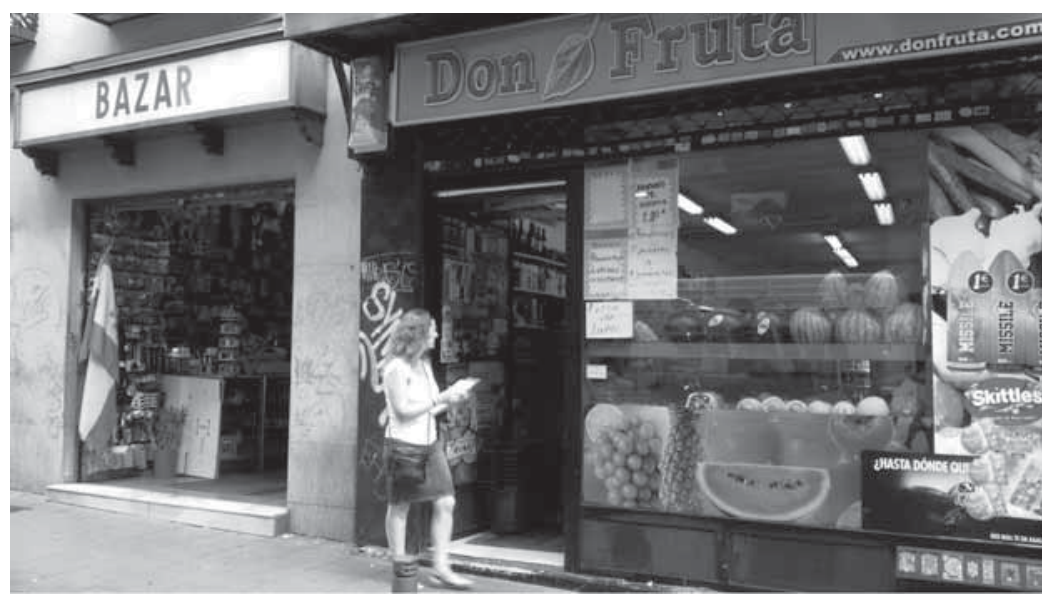

Fuente: elaboración propia.

La ropa, el calzado y los complementos constituyen un sector que, lógicamente, ha bajado mucho sus ventas en general. Muchos comercios han tenido que cerrar. Pero los de titularidad china, por el contrario, han aumentado bastante en los últimos años, con lo cual han contrarrestado los cierres de autóctonos y de emprendedores de otras nacionalidades. La estrategia comercial de los comerciantes chinos del sector de la confección ha consistido principalmente en mantener unos precios bajos, pero vender una moda muy occidental y orientada a la población más joven. Se ha pasado de la venta de ropa fuera de moda en bazares, a la ropa juvenil de temporada en tiendas especializadas, pero manteniendo una política de precios muy moderados.

El sector de servicios que más auge ha experimentado en estos tiempos de crisis es el de peluquería y estética. Estos negocios requieren muy poca inversión en los locales, la adecuación de los mismos es mínima y el gasto en los productos que usan tampoco es excesivo. La mano de obra es mayoritariamente oriental y latinoamericana; acepta bajos salarios o, si se trata de los propios emprendedores, se conforma con menores beneficios empresariales. 
"A mi peluquería antes casi no venían españolas, pero con la crisis vienen más, porque ésta es más barata”. (Empresaria cubana)

Los bazares y en general las antiguas tiendas de todo a cien comercializan cada vez más productos cotidianos (limpieza, mercería, utensilios de cocina, etc.) y reducen la oferta en regalos y productos superfluos, menos demandados en tiempos de crisis. A los emprendedores orientales se han unido los de otras nacionalidades, con productos más atractivos para la población occidental y en general de mayor calidad, aunque baratos.

"El dueño del bazar es argentino. A mí me paga 450 euros al mes. Vivo muy cerca, mi pareja trabaja y asi nos mantenemos". (Empleado boliviano)

Los empresarios inmigrantes que se especializaron en la venta de productos que consideraban símbolos de su identidad nacional se localizaron básicamente en áreas donde residían muchos de sus compatriotas, pero este tipo de negocios, marcadamente étnicos, ha ido disminuyendo con la crisis económica. En cambio, se han mantenido e incluso han aumentado las tiendas de inmigrantes en las que, gracias a la importación y, eventualmente, a la fabricación en talleres más o menos legales y con menores costes de producción, se ofrecen productos a precios más económicos que los autóctonos (tiendas de ropa, bazares, tiendas de electrodomésticos, etc.). Estos empresarios se abastecen en almacenes de importación mayorista de una gran variedad de productos fabricados en China de tipo low cost. Estos mayoristas suelen concentrarse en determinados polígonos, como el conocido Cobo Calleja en Fuenlabrada.

Hay también modas imperantes en cuanto al tipo de negocios establecidos. Así, hace unos años se abrieron numerosos locutorios que hoy en día han cerrado o han diversificado sus servicios. En muchos locutorios se han añadido otros servicios, como la venta de productos propios de los países de origen de los dueños de estos establecimientos.

En la foto (Figura 3) que mostramos a continuación puede observarse cómo el locutorio también anuncia que vende y repara teléfonos móviles.

En el comercio chino, que es el predominante, también se ven estos cambios de tendencia, los bazares y las tiendas de alimentación se han estancado, sobre todo los primeros, a favor de las tiendas 
de ropa y complementos de fabricación china, montadas de forma más moderna. Y actualmente se observa gran cantidad de nuevas aperturas de negocios de manicura y tratamientos de belleza.

\section{FIGURA 3}

PASEO DE EXTREMADURA EN EL DISTRITO DE LATINA

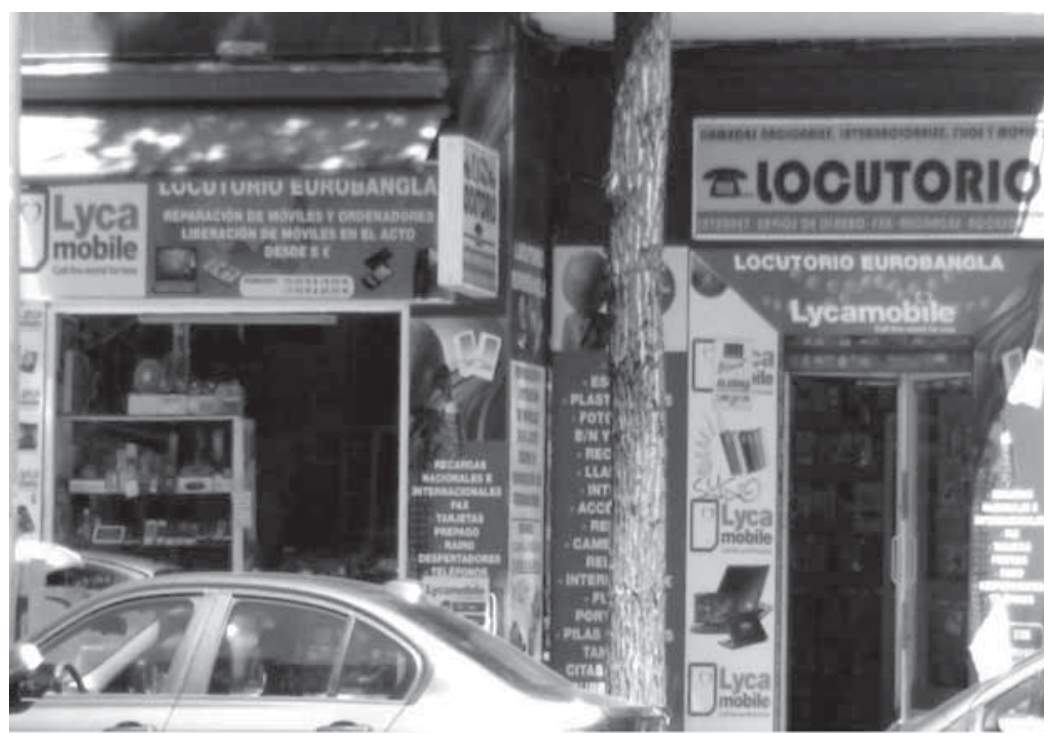

Fuente: elaboración propia.

\section{NACIONALIDADES PREDOMINANTES: ANTES Y DESPUÉS}

La notable variedad de procedencias de emprendedores inmigrantes que registramos en 2009, ha dado paso en la actualidad a un espectro mucho más reducido que se caracteriza por el aumento del empresariado chino y el descenso de los empresarios de origen latinoamericano (Cuadro 5).

Esta disminución de los emprendedores de Latinoamérica está en consonancia con el descenso general de inmigrantes latinoamericanos en España (García Ballesteros et al., 2014). 
Los ciudadanos procedentes de China, en cambio, son los emprendedores predominantes. Se organizan formando auténticas cadenas en expansión, con una clara tendencia a desplazarse de la hostelería a un comercio cada vez más diversificado. (Beltrán y Sáiz, 2013, 2015). Su clientela siempre ha tendido a ser general y no coétnica (Beltrán y Sáiz, 2009; Betrisey, 2010), salvo en los barrios de fuerte concentración de residentes chinos, principalmente en el distrito de Usera (Tébar, 2010; 2013)

El número de autónomos chinos ha crecido un 74\% en la región desde 2008 a 2014, según los datos publicados por el Ministerio de Empleo y Seguridad Social. En 2014, en la Comunidad de Madrid había un total de 11.374 autónomos chinos, 4.843 más que en el año 2008. Los empresarios chinos constituyen una fuente importante de empleo para sus compatriotas (Betrisey, 2010).

Un aspecto interesante que se ha detectado en el trabajo de campo es la diferenciación existente entre los chinos llegados a España y su segunda generación, sus integrantes tienen la nacionalidad española, hablan bien nuestro idioma y, los que siguen la tradición comercial, organizan sus negocios prescindiendo esencialmente de cualquier elemento decorativo oriental.

\section{CuAdro 5}

PROCEDENCIA DE LOS EMPRENDEDORES

\begin{tabular}{|l|c|c|}
\hline \multicolumn{1}{|c|}{$\begin{array}{c}\text { Zona geográfica de procedencia de los } \\
\text { emprendedores }\end{array}$} & $\begin{array}{c}\text { \% sobre el total } \\
\mathbf{2 0 0 9}\end{array}$ & $\begin{array}{c}\text { \% sobre el total } \\
\mathbf{2 0 1 5}\end{array}$ \\
\hline China & 42 & 47 \\
\hline Países de América Andina & 36 & 32 \\
\hline Países del Caribe & 7 & 3 \\
\hline Países del Norte de África & 4 & 7 \\
\hline Países Indostánicos & 3 & 2 \\
\hline Países del Cono Sur & 3 & 1 \\
\hline Países de Europa Del Este & 2 & 1 \\
\hline Países de África Subsahariana & 1 & 1 \\
\hline Países de Extremo Oriente & 1 & 1 \\
\hline Países de Oriente Próximo & 1 & 100 \\
\hline TOTAL & 100 & \\
\hline
\end{tabular}

Fuente: elaboración propia. 
En un segundo puesto se sitúan los empresarios de origen latinoamericano. Destacan ecuatorianos, colombianos, peruanos, argentinos, cubanos y dominicanos, algunos con el beneficio de la nacionalidad española. En el caso de los latinoamericanos la presencia de mujeres emprendedoras es más frecuente que entre el empresariado de otras nacionalidades (Oso y Ribas, 2004; Oso y Villarés, 2005; Jiménez Blasco y Redondo, 2010), y se ha acentuado en los últimos años por el auge de los negocios de peluquería y estética que son montados en su mayoría por mujeres. En el sector de la hostelería se sigue observando un cierto peso de los empresarios procedentes de países del Cono Sur (García Ballesteros y Jiménez Blasco, 2012).

Como muestra el Cuadro 5 los emprendedores procedentes de Bangladesh, Sri Lanka, India y Pakistán han aumentado significativamente. Su orientación comercial está bastante diversificada: alimentación, bazares, telefonía, estética y hostelería. En menor medida se dedican a la ropa, calzado y complementos.

"Llevo en España 12 años. Hace seis meses que monté este negocio (manicura y estética) y me va bastante bien”. (Emprendedora india)

Respecto a otras nacionalidades, se puede mencionar la presencia de marroquíes en negocios destinados en principio a sus compatriotas (carnicerías halal, por ejemplo), pero que buscan ampliar su clientela al resto de la población, comportándose como un comercio de proximidad.

En los distintos ejes comerciales analizados se observan algunas diferencias en cuanto a las nacionalidades presentes en los mismos, aunque es claro el predominio de las personas procedentes de China y de países como Colombia, Perú y Ecuador, si bien ha habido una bajada en el número de emprendedores de estos tres últimos países, a la par que se ha producido un ascenso importante de los empresarios originarios de países indostánicos (Beltrán et al., 2006).

En el caso de los naturales de África es destacable su concentración en áreas reducidas, lo que contrasta con la dispersión, por ejemplo, de los latinoamericanos, salvo el caso de los dominicanos que, en ocasiones, establecen diversos negocios muy próximos entre sí en zonas muy concretas, a las que se llega a denominar como "Pequeños Caribes". 


\section{FigURA 4}

\section{CALLE DEL GENERAL RICARDOS EN EL DISTRITO DE CARABANCHEL}

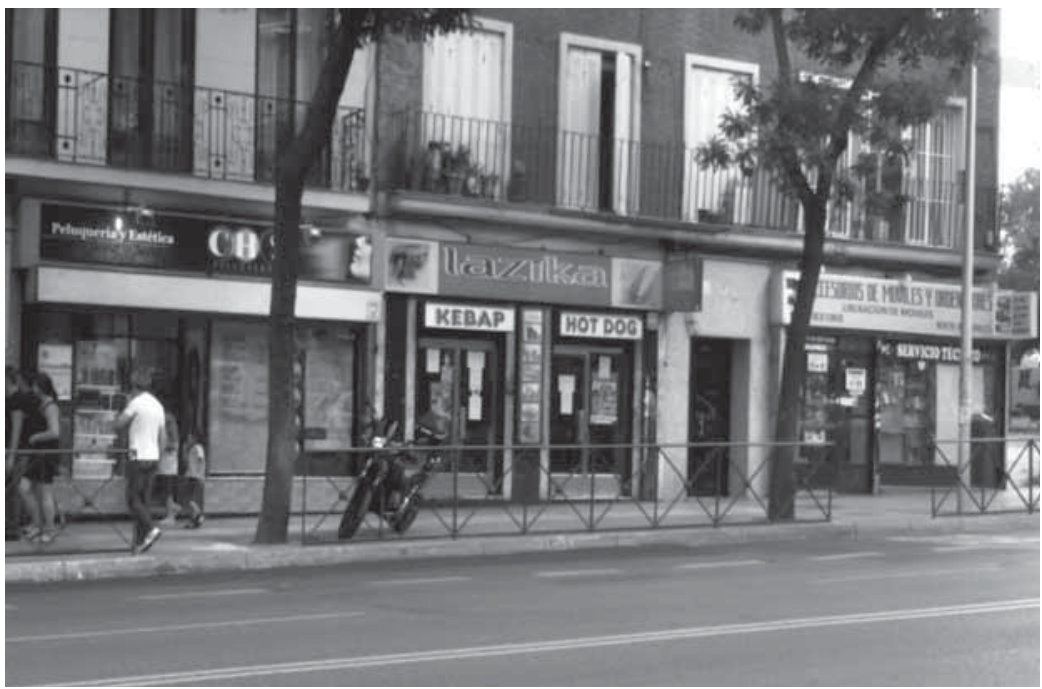

Fuente: elaboración propia.

\section{ESTRATEGIAS COMERCIALES Y ESPACIALES DEL EMPRESARIADO INMIGRANTE ADOPTADAS DURANTE LA CRISIS ECONÓMICA}

Un análisis de la localización de la población extranjera afiliada al comercio en los distintos barrios madrileños indica una cierta correspondencia entre las mayores concentraciones de inmigrantes y las de los comercios regentados por ellos o en los que trabajan. Esta correlación entre inmigrantes residentes y número de establecimientos regentados por inmigrantes se constató con los datos proporcionados por el Ayuntamiento de Madrid, correspondientes al año 2010 (García Ballesteros et al. 2012b). En este trabajo, sus autores mostraron que existían correlaciones elevadas positivas (coeficiente de correlación lineal de Pearson por encima de 0,7) en los barrios de los distritos de Centro, Arganzuela, Tetuán, Latina, Carabanchel, Usera, Villaverde y Puente de Vallecas (Figura 5). No se obtuvieron, sin em- 
bargo, valores tan altos de correlación en otros distritos, sobre todo, porque los datos medios por barrios encubren una gran diversidad espacial, desde un punto de vista socioeconómico y urbanístico. Un análisis más desagregado territorialmente, por ejemplo, por secciones censales, hubiera mostrado mucho mejor las correspondencias entre áreas de inmigrantes residentes y afiliados extranjeros en el "Comercio". No se han podido calcular las correlaciones en fechas más recientes por no disponer de los datos necesarios para ello con posterioridad al año 2010.

\section{FIgURA 5}

DISTRITOS DE LA CIUDAD DE MADRID CON ALTA CORRELACIÓN POSITIVA ENTRE LA PROPORCIÓN DE RESIDENTES EXTRANJEROS Y EL NÚMERO DE ESTABLECIMIENTOS REGENTADOS POR INMIGRANTES (2010)

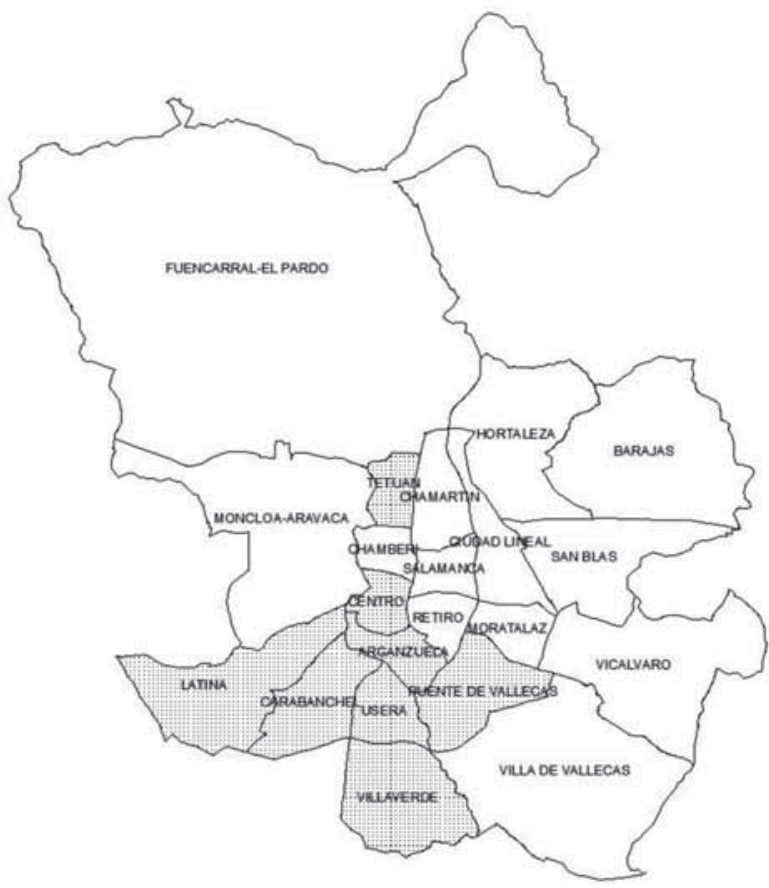

Fuente: Banco de Datos. Ayuntamiento de Madrid. Elaboración propia. 
A pesar de la cierta asociación existente entre inmigrantes residentes y concentración de negocios étnicos, hay que señalar una tendencia clara a su dispersión por toda la ciudad, pudiéndose observar comercios y servicios de inmigrantes en todos los barrios de Madrid, tanto céntricos como periféricos, y de muy distintos contenidos sociales.

Las zonas que muestran una fuerte concentración de negocios detentados por inmigrantes de una misma nacionalidad se convierten, con frecuencia, en áreas de encuentro para sus compatriotas, como ocurre en el llamado "gueto dominicano en Madrid", en torno a las calles de Almansa, Topete y Tenerife, muy próximas al tramo de la calle Bravo Murillo analizado en este artículo (García Escalona, 2010). Igualmente sucede en Usera, donde se han establecido multitud de establecimientos chinos, mayoritariamente frecuentados por la propia comunidad china residente en Madrid. Estos negocios muestran su publicidad y letreros en chino (Tébar, 2010, 2013).

Los clientes entrevistados afirman que las tres razones principales por las que acuden a los negocios de inmigrantes son: sus precios más bajos, su amplio horario de apertura, y el cierre de gran parte de los comercios tradicionales de la zona. Los consumidores inmigrantes nos indican, en un buen número de casos, que prefieren comprar en establecimientos de inmigrantes, sobre todo, si son de compatriotas, por razones de ayuda muta.

La mayor diferencia que observamos a partir de la comparación de las entrevistas que se hicieron en el año 2009 y las del año 2015 es que la proporción de clientes españoles ha aumentado significativamente. En el año 2009 se señalaba con frecuencia que los negocios de inmigrantes tenían un aspecto descuidado, productos de peor calidad, ausencia de marcas conocidas... Mientras, en las entrevistas realizadas en el año 2015 se hace una valoración general más positiva de este tipo de negocios.

La localización de negocios de inmigrantes en los ejes comerciales de las zonas estudiadas difiere un poco de unas a otras. En el paseo de Extremadura, la calle de General Ricardos (Figura 4) o la Avenida de la Albufera muchos negocios han ido cerrando por jubilación de sus propietarios y falta de relevo generacional. Los locales que han ido cerrando, de coste razonable, han sido arrendados por inmigrantes extranjeros para establecer "comercios de proximidad" y negocios con un doble tipo de clientela: los inmigrantes que viven en la zona 
y la población española residente en la misma que tiene menor movilidad y menos recursos económicos, en general personas mayores.

En cambio, otros ejes comerciales, como las calles de Bravo Murillo y Alcalá, muestran un comercio más especializado con un área de influencia superior. Los negocios regentados por inmigrantes conviven con franquicias y tiendas de moda joven establecidas en casi todas las zonas comerciales de Madrid. Estos ejes tienen tramos en los que los negocios de inmigrantes son escasos por la casi nula disponibilidad de locales, sin embargo, en otros tramos de dichos ejes, menos acaparados por conocidas firmas comerciales, sí los encontramos.

En los barrios céntricos de Madrid el comercio tradicional antes de la década de los 80 del siglo XX era mayoritariamente de venta de productos de uso cotidiano con clientela fija, residente en la zona (Cebrián y Bodega, 2002). En las calles más comerciales del distrito Centro, como podría ser el caso de la calle Hortaleza, coexistían tiendas de barrio con comercios más especializados con un área de influencia que abarcaba toda la ciudad (Cámara de Comercio e Industria de Madrid, 1983).

El comercio tradicional en estos barrios, con numerosos edificios viejos, obsoletos y deteriorados, entró en crisis, sobre todo, a partir de los años 80 (Jiménez Blasco, 2007).

Los nuevos negocios de inmigrantes por un lado suplen esta falta del comercio tradicional del barrio. Por ejemplo, las pequeñas tiendas de alimentación sustituyen a los antiguos ultramarinos y los bazares venden muchas de las cosas que antes se compraban en droguerías, mercerías, papelerías, etc. que han ido desapareciendo con el paso de los años. El problema está en que los estándares de calidad de muchos de estos nuevos negocios, sobre todo de los que se dedican a productos cotidianos, suelen ser menores, y el aspecto de los locales está descuidado en muchas ocasiones. Esto conduce a que, a pesar de su efecto en la revitalización del tejido comercial del barrio, éste sea percibido por los vecinos de siempre como más deteriorado que antes.

\section{CONCLUSIONES}

Los inmigrantes que decidieron establecer un negocio en la ciudad de Madrid han intentado continuar en la actividad empren- 
dida, restringiendo sus ganancias y modificando su oferta para adaptarse mejor a los tiempos de crisis. Han seguido teniendo una presencia general en toda la ciudad, no hay barrios que no cuenten con negocios de inmigrantes. Eso sí, de unos barrios a otros cambia considerablemente la densidad, la tipología y la calidad de los negocios, así como las nacionalidades predominantes de los empresarios extranjeros.

No obstante, sigue habiendo una mayor concentración de negocios de empresariado inmigrante en los barrios en los que residen en mayor proporción los inmigrantes empadronados en la ciudad de Madrid. Estas zonas resultan más atractivas para los emprendedores inmigrantes, porque cuentan con una probable mayor clientela de compatriotas, y porque los locales comerciales tienen precios más asequibles. Aunque, a la vez, es cierto que el cliente inmigrante, muy afectado por el desempleo, ha reducido mucho su capacidad de consumo. Esto ha llevado a algunos negocios que estaban muy enfocados a la población inmigrante a cerrar o cambiar su estrategia y orientarse también a la población autóctona.

En el trabajo de campo llevado a cabo en el año 2009, todavía en los momentos iniciales de la crisis económica, se apreciaba en determinadas calles una concentración de negocios de emprendedores de la misma nacionalidad, con clientela prácticamente de compatriotas y con una finalidad social añadida a la laboral y económica, pues se habían convertido en lugares de reunión y mutua ayuda. La realidad actual es muy distinta.

En todas las calles estudiadas ha habido cambios significativos en el tipo de servicios y productos ofrecidos, adaptándose en un tiempo record a la demanda existente. La calle de Hortaleza y el paseo de Extremadura, dentro de los ejes comerciales estudiados en este trabajo, son los que más han cambiado.

En el momento actual los comerciantes extranjeros muestran cada vez mayor interés en la venta de productos de uso cotidiano y bajo precio, que no exijan grandes desembolsos económicos (alimentación, droguerías, etc.), habiendo descendido el número de comercios de productos superfluos (bisutería, bazares, etc.). Son continuos los cierres y aperturas, pues la inversión en los locales es exigua y mayoritariamente son arrendados. Si no se encuentra una rentabilidad en poco tiempo, no se continúa con el negocio. 
En cuanto a los locales destinados a servicios también se siguen prefiriendo aquellos que no requieren fuertes inversiones (locutorios, peluquerías, estética), sin olvidar una marcada tendencia hacia la hostelería. En este campo empresarial los inmigrantes comenzaron a montar pequeños bares y restaurantes, así como negocios del tipo kebabs y comidas preparadas que tenían asegurada la clientela con trabajadores que tienen poco tiempo para comer y con jóvenes que suelen consumir este tipo de comidas durante su tiempo de ocio. Lógicamente este tipo de negocios es más habitual en zonas céntricas, próximas a oficinas y a locales de ocio juvenil. En el trabajo de campo hemos comprobado que estos negocios no han sufrido demasiado los efectos de la crisis económica, pues se sigue manteniendo un número de locales similar desde los inicios de la misma.

Se constata un relativo receso en la actividad empresarial de los inmigrantes de origen latinoamericano, al menos desde hace un par de años. El tipo de negocio de este colectivo de emprendedores, caracterizado por una oferta más enfocada hacia sus propios compatriotas, ha sufrido más las consecuencias de la contracción del consumo, debido al descenso del número de inmigrantes de origen latinoamericano y a la fuerte disminución de la capacidad adquisitiva de los que permanecen en España.

Pero, en general, incluyendo a los empresarios inmigrantes de todas las nacionalidades, ha habido, más que un descenso del número de establecimientos, una adaptación de la oferta a los cambios en la demanda, que se deben no solo a los efectos de la crisis económica, sino también a modificaciones en los hábitos de consumo de los madrileños, tema sobre el que debemos profundizar en estudios posteriores.

En definitiva, la presencia de pequeños negocios de inmigrantes en la ciudad de Madrid es un fenómeno que ha ido en aumento desde hace unos veinte años, si bien experimentó un cierto descenso entre los años 2008 y 2010, se ha ido recuperando poco a poco a partir de esta fecha. Y se puede afirmar que el trabajo autónomo en el sector comercial y de servicios en la ciudad de Madrid sigue siendo una opción para un buen número de inmigrantes, quienes lo consideran como un medio de ascenso social y de mejora profesional, tras haber sido previamente muchos de ellos trabajadores por cuenta ajena. 


\section{BIBLIOGRAFÍA}

Ambrosini, M. (1996): “Ethnicité et marché du travail: les immigrés dans le système économique italien”. Sociologia del Lavoro, n 66-67, pp. 307-327.

Aramburu, M. (2002): "Los comercios de inmigrantes extranjeros en Barcelona y la recomposición del "inmigrante" como categoría social". Scripta Nova, Revista electrónica de Geografía y Ciencias Sociales, Vol. VI, n 108.

ArJona, A. y Checa, J. C. (2005): “Emprendedores étnicos en Almería”. Sociología del Trabajo, no 54, pp. 101-125.

- (2006): "Economía étnica: teorías, conceptos y nuevos avances", Revista Internacional de Sociología, Vol. LXIV, no ${ }^{\circ}$, pp.117-143.

- (2007): "Ubicación espacial de los negocios étnicos en Almería. ¿Formación de enclaves económicos étnicos?” Estudios Geográficos, LXVIII, 263, pp. 391-415.

Beltrán, J.; Betrisey, D.; LóPez, A. (2006): Población y actividades económicas de las comunidades asiáticas en España. Barcelona, Fundación CIDOB.

Beltrán, J., Oso, L. y Ribas, N. (Coords.) (2006): Empresariado étnico en España. Madrid. Observatorio Permanente de la Inmigración y Fundación CIDOB.

Beltrán Antolín, J. y Sáiz López, A. (Eds.) (2009): Empresariado asiático en España. Barcelona. Fundación CIDOB.

Beltrán Antolín, J. y SÁiz López, A. (2013): "Del restaurante chino al bar autóctono. Evolución del empresariado de origen chino en España y su compleja relación con la etnicidad”, en M. Barros Nock y H. Valenzuela García (Eds.), Retos y estrategias del empresariado étnico. Estudios de caso de empresarios latinos en los Estados Unidos y empresarios inmigrantes en España. México, D.F, Centro de Investigaciones y Estudios Superiores en Antropología Social (CIESAS), pp. 85-108.

- (2015): “A contracorriente. Trabajadores y empresarios chinos en España ante la crisis económica (2007-2013)”. Migraciones, Revista del Instituto Universitario de Estudios sobre Migraciones, $\mathrm{n}^{\circ} 37$, pp. 125-147.

Betrisey Nadali, D. (2010): “Empresarios y 'líderes' chinos en Madrid. Prácticas políticas y económicas”. Revista CIDOB d'Afers Internacionals, $\mathrm{n}^{\circ}$ 92, pp. 207-222.

BucKley, M. (1998): “Inmigración y comercio en Madrid. Nuevos negocios para nuevas gentes". Anales de Geografía de la Universidad Complutense, $\mathrm{n}^{\circ} 18$, pp. 286-297.

CÁmara de Comercio E Industria de Madrid (1983): Establecimientos tradicionales madrileños, A ambos lados de la Gran Vía. Madrid.

Cavalcanti, L. (2007): "Negocios étnicos: importación y repercusiones de una categoría”, E. Santamaría (Ed.): Los retos epistemológicos de las migraciones transnacionales. Barcelona, Ed. Antrophos.

- (2009): "Los tipos de iniciativas empresariales de los inmigrantes en las ciudades de Barcelona, Madrid y Valencia”. Revista Internacional de Organizaciones, $\mathrm{n}^{\circ} 2$, pp. 51-62. 
Cebrián de Miguel, J. A. y Bodega Fernández, I. (2002): "El negocio étnico, nueva fórmula de comercio en el casco antiguo de Madrid: el caso de Lavapiés”. Estudios geográficos, Vol. 63, pp. 559-580.

Cebrián, J. A.; Bodega, I. y Bordonado, J. (2004): “La iniciativa empresarial del inmigrante". Revista Economistas, n ${ }^{\circ}$ 99, pp. 116-122.

Cebrián, J. A.; Jiménez Blasco, B. C. y Resino, R. M. (2016): “Cambios recientes en los negocios de inmigrantes", conferencia pronunciada el 25 de febrero de 2016 en las VI Jornadas de Investigación en Geografía, Universidad Complutense de Madrid, en prensa.

Cohen, M. (2003): "Les commerçants chinois du quartier Popincourt", Panoramiques. Paris, Éditions Corlet Marianne, pp. 77-87.

Espinosa, A. (2008): “Comercio, centros históricos e inmigración”. Valero Escandell (Ed.): La inmigración en los centros históricos, publicaciones de la Universidad de Alicante, pp. 119-132.

- (2011): "El comercio étnico", Open Course Ware, material docente audiovisual de la asignatura "Geografía del Comercio y los Servicios" de la Universidad de Alicante. Accesible en: http://hdl.handle. net/10045/16765.

Escudero Gómez, L. A. (2008): Los centros comerciales. Espacios postmodernos de ocio y consumo. Cuenca, Ediciones de la Universidad de CastillaLa Mancha.

Evans, M. (1989): "Immigrant entrepreneurship: Effects of Ethnic Market Size and Linguistically Isolated Labor Pools". American Sociological Review, no54, p. 950-962.

Galbraith, C.; Rodriguez, C. y Stiles, C. (2007): "Ethnic economies, social capital and the economic theory of the clubs". Leo Paul Dana (Ed.). Handbook of research on ethnic minority entrepreneurship, Edward Elgar Publishing Limited, pp. 16-29.

GARCÉs, A. (2011): “Comercio inmigrante y economías étnicas: síntesis y críticas de los debates vigentes", Polis. 29.

García Ballesteros, A.; García Escalona, E.; Hernando F.; Jiménez Blasco, B. C.; Mayoral, M. (2004): Inmigración y Sistema Productivo en la Comunidad de Madrid. Comunidad de Madrid, Consejería de Economía e Innovación Tecnológica.

García Ballesteros, A. (2005): "La inserción laboral de los inmigrantes extranjeros como trabajadores autónomos en la Comunidad de Madrid". Barómetro de Economía de la Ciudad de Madrid, pp. 99-107.

García Ballesteros, A.; García Escalona, E.; Hernando F.; Jiménez Blasco, B. C. y Mayoral, M. (2006): Inmigrantes emprendedores en la Comunidad de Madrid. Comunidad de Madrid, Consejería de Economía e Innovación Tecnológica.

García Ballesteros, A. Hernando Sanz, F. y Jiménez Blasco, B. C. (2012a): “El empresariado étnico en la ciudad de Madrid. Análisis de sus trayectorias a través de estudios de caso", Mourad Aboussi (Coord): El codesarrollo a debate. Granada, Editorial Comares, pp. 125-136. 
García Ballesteros, A.; Jiménez Blasco, B. C.; Mayoral, M. y Viñas, G. (2012b): "La actividad empresarial de los inmigrantes emprendedores en la comunidad de Madrid” en Empresariado inmigrante, instituciones y desarrollo. Granada, Ed. Comares, pp. 113-130.

García Ballesteros, A.; Jiménez Blasco, B. C. (2012c): "Immigrant Business Strategies in the City of Madrid". International Journal of Human and Social Sciences, Vol. 2, $\mathrm{n}^{\circ}$ 20, pp. 86-97.

García Ballesteros, A.; Jiménez Blasco, B. C. y Mayoral Peñas, M. M. (2014): “Emigración de retorno y crisis en España”. Scripta Nova, 2014. Vol. XVIII, n 491.

García Escalona, E. (2010): "Viajar por Madrid: nuevos rostros y voces”. Ángulo Recto. Revista de estudios sobre la ciudad como espacio plural, Vol. 2, $\mathrm{n}^{\circ} 2$, pp. 83-101.

Gil-Alonso, F. y Vidal-Coso, E. (2015): “Inmigrantes extranjeros en el mercado de trabajo español: ¿más resilientes o más vulnerables al impacto de la crisis? Migraciones, Revista del Instituto Universitario de Estudios sobre Migraciones, $\mathrm{n}^{\circ}$ 37, pp. 97-123.

Gómez Crespo, P. (2006): Inmigración y comercio en Madrid: empresarios, consumidores, trabajadores y vecinos. Observatorio de las Migraciones y de la Convivencia Intercultural de la ciudad de Madrid. Colección Monografías, 1.

Grupo de Investigación de la Universidad Complutense “Geografías de la INMigración EN la COMUNIDAD DE MADRID” (2010): Efectos de la presencia de la población inmigrante no comunitaria en el tejido comercial de la ciudad de Madrid. Informe realizado para el Área de Economía y Empleo del Ayuntamiento de Madrid.

Herranz Gómez, Y. (1991): "Un pequeño empresariado latinoamericano en Madrid”,. Sociología del Trabajo, no 13, pp. 75-95.

Jiménez Blasco, B. C. (2007): "Mujer y ciudad: cambio social y nuevos espacios urbanos”. En Segura Graíño, C. (Coord.), Mujeres y espacios urbanos. Homenaje a Christina de Pizan en el VI Centenario de la 1 edición de "La ciudad de las damas", 1405-2005, Colección Laya, n² 27, pp. 169-174.

Jiménez Blasco, B. C.; Mayoral Peñas, M. y Martín Jiménez, A. (2008): “Los negocios de los inmigrantes latinoamericanos en Madrid”, en F. J. Antón Burgos y S. Sánchez Moral (Eds.), Comercio, servicios y transporte. Patrones de una sociedad avanzada. Grupo de Geografía de los Servicios. Asociación de Geógrafos Españoles, pp. 215-226.

Jiménez Blasco, B. C. y Redondo González, A. (2010): “Condiciones, laborales, familiares y sanitarias de las mujeres inmigrantes en la ciudad de Madrid”, en Calvo Buezas, T. y Gentil García, I. (Eds.), Inmigrantes en Estados Unidos y en España. Protagonistas en el siglo XXI. CEMIRA, pp. 623-637.

Kloosterman, R. (2001): "Immigrant Entrepreneurship and the institutional Context: A Theoretical Exploration”, en J. Rath (Ed): Immigrant Business. The economic, politic and social environtment. Centre for Research in Ethnic Relations, Warwick. 
Light, I. y Bнаснu, P. (Eds.) (1992): Immigration and Entrepreneurship: Culture, Capital and Ethnic Networks. New Brunswick,Transaction Publishes.

Light, I. y Bonacich, E. (1988): Immigrant Entrepreneurs. Koreans in Los Angeles 1965-1982. Los Angeles, University of California Press.

Light, I. y Gold, S. (2000): Ethnic Economies. San Diego, EE.UU., Academic Press.

Miret, N. y Serra del Pozo, P. (2013): “El papel de la inmigración extranjera en el cambio social y urbano de el Besòs i el Maresme, un barrio periférico de Barcelona. Interrogaciones a partir de un estudio exploratorio". Estudios Geográficos, Vol. 74, nº 274, pp. 193-229.

Muñoz-Bullón, F. (2014): "Emprender en tiempos de crisis: la actividad emprendedora de los inmigrantes en España”. Cuadernos Económicos de ICE, $\mathrm{n}^{\circ} 87$, pp. 127-160.

Oso, L. y Ribas, N. (2004): Empresariado étnico y género: dominicanas y marroquies en Madrid y Barcelona. Ponencia presentada en el IV Congreso sobre la Inmigración en España, Girona, 10-13 noviembre.

Oso, L. y Villares, M. (2005): “Mujeres inmigrantes latinoamericanas y empresariado étnico: dominicanas en Madrid, argentinas y venezolanas en Galicia”. Revista Galega de Economía, Vol. 14, n 1-2, pp. 261-278.

Pagliarin, S. (2011): "Empresariado étnico y formación de enclaves comerciales: el papel de las redes sociales en el caso de la calle de Sant Pau en Barcelona”, Scripta Nova, Vol. XVII, nº 962.

Parella, S. (2006): "Estrategias de los comercios étnicos en Barcelona, España”. Política y Cultura, n ${ }^{\circ} 23$, pp. 257-275.

Portes, A; Guarnizo, L. y Landolt, P. (2003): La globalización desde abajo: transnacionalismo inmigrante y desarrollo. La experiencia de Estados Unidos y América Latina. México, Facultad Latinoamericana de Ciencias Sociales.

Portes, A. y Shafer, S. (2006): "Revisiting the Enclave Hypothesis: Miami Twenty-Five Years Later". The Center for Migration and Development, Princeton University Working Paper Series 06-10.

RIESCO, A. (2003): "Enclaves y economías étnicas desde la perspectiva de las relaciones salariales”. Cuadernos de Relaciones Laborales, 21(2), pp. 103-125.

Serra del Pozo, P. (2006): El comercio étnico en el distrito de Ciutat Vella en Barcelona. Barcelona, Fundació La Caixa.

Solé, C.; Parella, S. (2005): Negocios étnicos. Los comercios de los inmigrantes no comunitarios en Catalunya. Barcelona, Fundación CIDOB.

Solé, C.; Parella, S. y CavalcantI, L. (2007): El empresariado inmigrante en España. Colección Estudios Sociales, $\mathrm{n}^{\circ} 21$. Fundación La Caixa.

Tébar Arjona, J. (2010): Mapa del Chinatown de Madrid. Madrid, Bubok Publishing S. L.

- (2013): "Patrones espaciales de la diáspora china en el mundo, España y Madrid”. Historia Actual Online, n 30, pp. 89-103.

Torres Pérez, F. (2006): "Las dinámicas de la convivencia en un barrio multicultural. El caso de Russafa (Valencia)”. Papeles del CEIC. Vol. 1. No. 23. 
Trinidad García, M. L. (2003): “El trabajo por cuenta propia de los extranjeros en España”. Migraciones, Revista del Instituto Universitario de Estudios sobre Migraciones, $\mathrm{n}^{\circ}$ 13, pp. 61-106.

Wilpert, C. (2003): “Germany: from workers to entrepreneurs”, en R. Kloosterman y J. Rath (Ed.), Immigrant entrepreneurs: venturing abroad in the age of globalization. Berg Publishers.

Wilson, K. y Portes, A. (1980): "Immigrant Enclaves: An Analysis of the Labor Market Experiences of Cubans in Miami”. American Journal of Sociology, no 86, pp. 295-319.

Zárate Martín, A. (2001): "Imágenes mentales del centro de Madrid, el barrio de Lavapiés”. Boletín de la Real Sociedad Geográfica, Tomo CXXXVII, pp. 137-184. 